\title{
Charge exchange nuclear excitations and beta decay within the self consistent deformed QRPA
}

\author{
Marco Martini * \\ Ghent University, B-9000 Gent, Belgium; CEA, DAM, DIF, F-91297 Arpajon, France; \\ Université Libre de Bruxelles, B-1050 Brussels, Belgium \\ E-mail: martini.marco@gmail.com
}

\section{Sophie Péru}

CEA, DAM, DIF, F-91297 Arpajon, France

E-mail: sophie.peru-desenfants@cea.fr

\section{Stephane Goriely}

Université Libre de Bruxelles, B-1050 Brussels, Belgium

E-mail: sgorielyeastro.ulb.ac.be

\begin{abstract}
Spin-isospin nuclear excitations play a crucial role in astrophysics where they govern beta-decay, electron and neutrino capture processes, hence stellar evolution and nucleosynthesis. These excitations are studied here within the fully self-consistent proton-neutron quasiparticle randomphase approximation using the finite-range Gogny interaction. Axially symmetric deformations are consistently taken into account, both in the description of the ground states and spin-isospin excitations. We focus in particular on the Gamow-Teller excitations. A comparison of the predicted strength distributions to the existing experimental data is presented and the role of nuclear deformation analyzed. The Gamow-Teller strength is used to estimate the beta-decay half-life of nuclei for which experimental data exist. A satisfactory agreement with experimental half-lives is found and justifies the additional study of the exotic neutron-rich $\mathrm{N}=82,126$ and 184 isotonic chains of relevance for the r-process nucleosynthesis. We also show the comparison between our theoretical predictions and the experimental half-lives for the much studied isotopic chains of Kr, $\mathrm{Sr}, \mathrm{Zr}$ and Mo which are strongly deformed.
\end{abstract}

XIII Nuclei in the Cosmos

7-11 July, 2014

Debrecen, Hungary

* M.M. acknowledges the Interuniversity Attraction Poles Programme initiated by the Belgian Science Policy Office (BriX network P7/12)

'Speaker.

${ }^{\ddagger}$ S.G. acknowledges the financial support of the F.N.R.S. 


\section{Introduction}

Spin-isospin nuclear excitations, in particular the Gamow-Teller (GT) resonances, nowadays play a crucial role in several fields of physics. First in fundamental nuclear physics by providing information on the nuclear interaction, the equation of state of asymmetric nuclear matter as well as the nuclear skin thickness. Second, in astrophysics where they govern $\beta$-decay, electron and neutrino capture processes, hence stellar evolution and nucleosynthesis. Finally, in particle physics in connection with the evaluation of the $V_{u d}$ element and the unitarity of the Cabibbo-KobayashiMaskawa quark-mixing matrix, on the one hand, and with neutrino physics beyond the standard model, on the other hand.

Experimentally the spin-isospin nuclear excitations are studied via charge-exchange reactions, such as $(\mathrm{p}, \mathrm{n}),(\mathrm{n}, \mathrm{p}),\left(\mathrm{d},{ }^{2} \mathrm{He}\right),\left({ }^{3} \mathrm{He}, \mathrm{t}\right)$ or $\left(\mathrm{t},{ }^{3} \mathrm{He}\right)$ and $\beta$-decay measurements. In spite of the great effort and interest, the whole nuclear chart is still not experimentally accessible, so that for the exotic nuclei, one can rely on theoretical models only. In this context one of the most popular models is the so-called proton-neutron quasiparticle random-phase approximation (pnQRPA). For a reliable prediction of the spin-isospin nuclear excitations, especially for experimentally unknown nuclei, two main features of the theoretical model are in order: the possibility to deal with deformed nuclei and the use of a unique effective nuclear force. The term unique has two different meanings here. First of all it implies that the interaction is the same for all nuclei, second that the nuclear interaction used to describe the ground and excited states is the same; this latter property is usually referred as the self-consistency of the calculation. Despite the relatively large number of pnQRPA calculations, the number of models nowadays including both features remains small. Furthermore, even in the limited number of self-consistent calculations performed either with the zero-range Skyrme-type forces or in the relativistic mean field framework, there often remains a coupling constant, typically in the particle-particle channel, which is treated as a free parameter usually adjusted to $\beta$-decay half-lives or to the position of GT excitation energies. The possibility to take into account the nuclear deformation is also very important. The $\beta$-decay properties of exotic neutron-rich nuclei (in particular those of interest to the r-process nucleosynthesis [1]) as well as the nuclear matrix elements for the double $\beta$-decay have been shown to depend significantly on the deformation parameter [2]. Furthermore, deformed nuclei present a strong fragmentation in the response functions and different nuclear shapes can be experimentally distinguished.

Here, we present a fully self-consistent axially-symmetric-deformed pnQRPA calculation [3] without any additional parameters beyond those characterizing the effective nuclear force, namely the finite-range Gogny force within its two parametrizations, D1M [4] and D1S [5]. This work represents a transposition to the charge-exchange sector of the fully consistent axially-symmetricdeformed QRPA calculations with the Gogny force, first presented in Ref. [6] and devoted to the study of electromagnetic excitations in deformed nuclei [7,8].

Our approach is based on the pnQRPA on top of axially-symmetric-deformed Hartree-FockBogoliubov (HFB) calculations. The HFB equations are solved in a finite harmonic oscillator basis. As a consequence, the positive energy continuum is discretized. All HFB quasiparticle states are used to generate the two-quasiparticle (2-qp) excitations. This means that in principle our calculation can be performed without a cut in energy or in occupation probabilities. According to the symmetries imposed in the present axially-symmetric-deformed HFB calculations in even- 


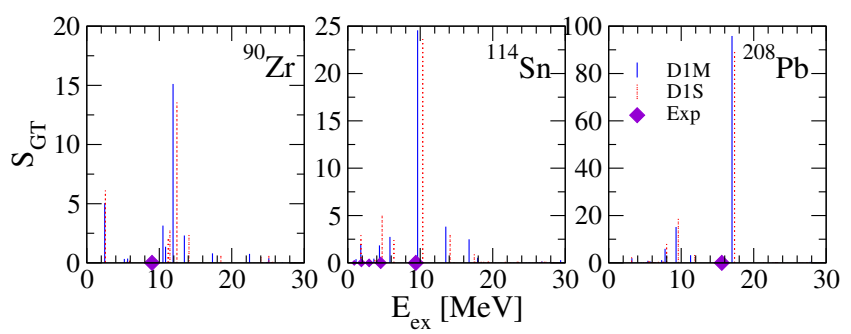

Figure 1: pnQRPA GT strength distributions in ${ }^{90} \mathrm{Zr},{ }^{114} \mathrm{Sn}$ and ${ }^{208} \mathrm{~Pb}$ calculated with the D1M and D1S forces. The experimental energy peaks obtained from scattering data $[9,10,11]$ are shown as diamonds on the $x$-axis.

even nuclei, the projection $K$ of the angular momentum $J$ on the symmetry axis and the parity $\Pi$ are good quantum numbers. Consequently, pnQRPA calculations can be performed separately in each $K^{\Pi}$ block. To solve the QRPA matrix equation we use the same numerical procedure recently applied to study the giant resonances of the heavy deformed ${ }^{238} \mathrm{U}$ nucleus [7]. It is based on a massive parallel master-slave algorithm. The solution of the pnQRPA matrix equation provides the energies $\omega_{\alpha, K}$ of the excited states of the parent nucleus and the set of amplitudes describing the wave function of the excited state in terms of the two quasiparticle excitations.

Once the pnQRPA matrix equation is solved, we can calculate the response to the GT operator

$$
\hat{O}_{G T}=\sum_{i=1}^{A} \vec{\sigma}(i) \tau_{-}(i)
$$

generating a spin-flip $(\Delta S=\Delta J=1)$ response. In an axially-symmetric-deformed nuclear system, the response function of a given $J^{\pi}$ contains different $K^{\pi}=0^{\pi}, \pm 1^{\pi}, \ldots, \pm J^{\pi}$ components. In this case the GT $J^{\pi}=1^{+}$distributions are obtained by adding twice the $K^{\pi}=1^{+}$result to the $K^{\pi}=0^{+}$ result. Details of how to go from the intrinsic to the laboratory frame can be found in Ref. [6].

\section{Results}

As a test case, we first consider the neutron closed-shell nuclei ${ }^{90} \mathrm{Zr}$ and ${ }^{208} \mathrm{~Pb}$, as well as the neutron open-shell nucleus ${ }^{114} \mathrm{Sn}$. Their GT strength distributions calculated with D1M and D1S interactions are shown in Fig 1. In the same figure, are shown the corresponding experimental values $[9,10,11]$ for the major excitation energies obtained from $(p, n)$ scattering data. The results are expressed as a function of the excitation energy $E_{e x}$ referred to the ground state of the daughter nucleus. In our model, it is obtained by subtracting a reference energy $E_{0}$ from the excitation energy $\omega_{\alpha, K}$ of the parent nucleus calculated in the pnQRPA, i.e., $E_{e x}=\omega_{\alpha, K}-E_{0}$. The reference energy corresponds to the lowest 2-qp excitation associated with the ground state of the odd-odd daughter nucleus in which the quantum numbers of the single quasi-proton and neutron states are obtained from the self-consistent HFB calculation of the odd-odd system. The two interactions give quite similar results for the position of the main peak. The D1M interaction is seen to give rise to a GT strength located at lower energies with respect to the one found with D1S. For the nuclei analyzed here, this energy shift rarely exceeds $0.5 \mathrm{MeV}$. As far as the comparison with experimental 


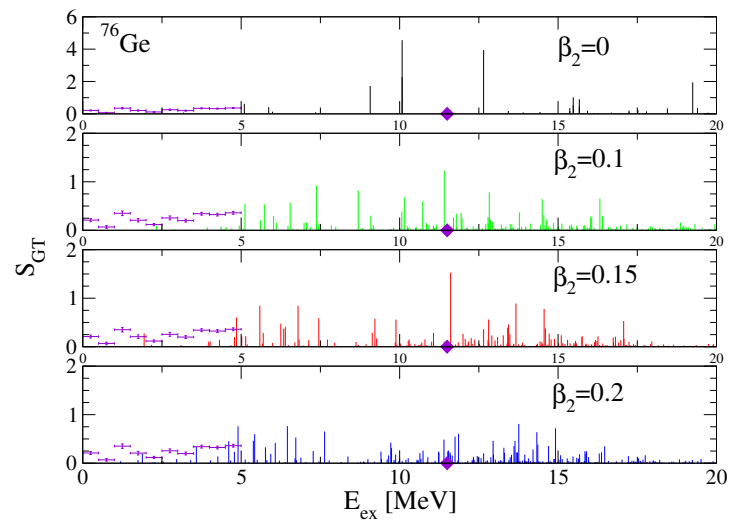

Figure 2: pnQRPA GT strength distributions in ${ }^{76} \mathrm{Ge}$ obtained with the D1M force for several values of the deformation parameter $\beta_{2}$, including the HFB ground state minimum at $\beta_{2}=0.15$. The experimental low-energy data [12] as well as the energy position of the main GT peak are also shown.

data is concerned, the agreement is seen to be rather satisfactory (Fig. 1). A small but systematic overestimate of the GT peak energy is found.

The above results refer to three spherical nuclei. As already emphasized, our approach describes axially symmetric deformed nuclei. As an example for a deformed nucleus, we consider ${ }^{76} \mathrm{Ge}$, a nucleus of particular interest in the neutrinoless double $\beta$-decay experiments. We show in Fig. 2 the ${ }^{76} \mathrm{Ge}$ GT excitations obtained with the D1M interaction for four different values of the quadrupole deformation parameter $\beta_{2}$, including the HFB minimum at $\beta_{2}=0.15$. As expected, the deformation tends to increase the fragmentation of the response. Calculations with different deformations produce peaks that are displaced. This is true not only for the giant resonance region but also for the low energy states. Recently the low energy part of the GT excitations of the ${ }^{76} \mathrm{Ge}$ nucleus has been studied with high precision [12] due to its importance for the neutrinoless double $\beta$-decay physics. We show this experimental result in Fig. 2 to compare to our results at different $\beta_{2}$. It appears that deformation effects influence the low-energy strength and that the spreading of the low-energy GT strength can be rather well reproduced for deformations around $\beta_{2}=0.15$, in contrast to what is found in the spherical approximation.

\section{Application to half-life calculations}

As a first application of our calculation, we now focus on the low-energy GT strength and more specifically on the $\beta^{-}$-decay half-lives. In the allowed GT decay approximation the $\beta^{-}$decay half-life $T_{1 / 2}$ can be expressed in terms of the GT strength function $S_{G T}$ according to

$$
\frac{\ln 2}{T_{1 / 2}}=\frac{\left(g_{A} / g_{V}\right)_{\mathrm{eff}}^{2}}{D} \int_{0}^{Q_{\beta}} f_{0}\left(Z, A, Q_{\beta}-E_{e x}\right) S_{G T}\left(E_{e x}\right) d E_{e x} .
$$

For the phase-space volume $f_{0}$ as well as the factor $D$ and the vector and axial vector coupling constants (including the quenching factor), we refer to the work of Ref. [13]. To estimate the $Q_{\beta}$ mass differences, we take experimental (and recommended) masses [14] when available or the D1M mass predictions [4], otherwise. 


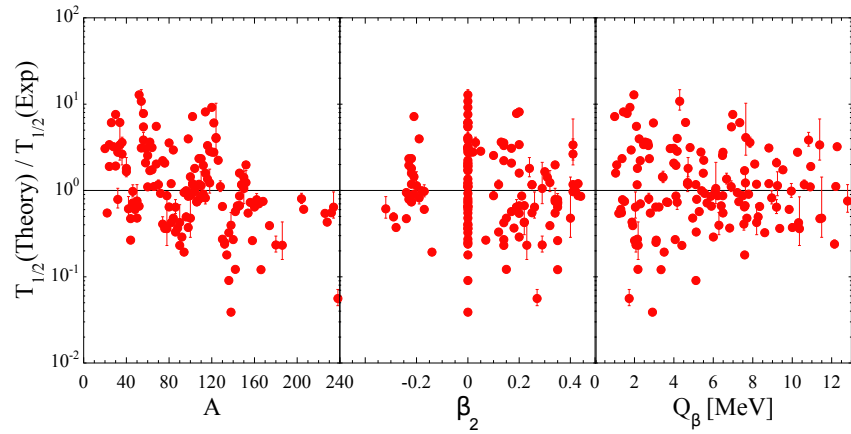

Figure 3: (color online) Ratio between the pnQRPA and experimental [15] $\beta^{-}$-decay half-lives as a function of $A, \beta_{2}$ and $Q_{\beta}$ for 145 even-even nuclei with an experimental halflife $T_{1 / 2} \leq 1000 \mathrm{~s}$.

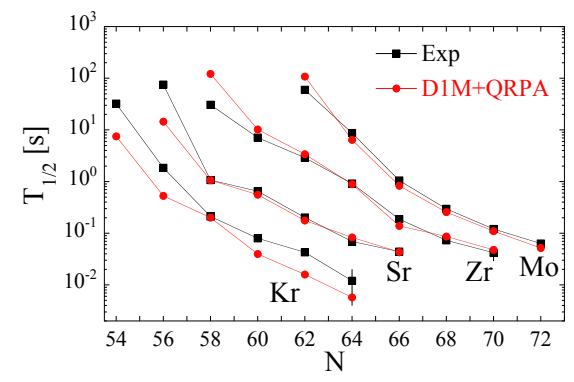

Figure 4: Comparison between experimental [15] and D1M+QRPA $\beta$-decay half-life predictions for the known isotopic chains of $\mathrm{Kr}, \mathrm{Sr}, \mathrm{Zr}$ and Mo.

The pnQRPA calculation provides, as shown in previous figures, a discrete strength distribution. In order to derive a smooth continuous strength function, the pnQRPA GT strength is folded with a Lorentz function, with a spreading width of $\Gamma=2 \mathrm{MeV}$, as classically done.

To give an idea of the global predictions of our model, we compare in Fig. 3 for even-even nuclei the pnQRPA (obtained with the D1M interaction) $\beta^{-}$-decay half-lives with the experimental data [15]. The results are plotted as a function of the mass number $A$, the deformation parameter $\beta_{2}$ and the $Q_{\beta}$ value. They turn out to be quite homogeneous with respect to $\beta_{2}$. Larger deviations are found for nuclei close to the valley of $\beta$-stability (Fig.3, right panel), i.e for low- $Q_{\beta}$ values, as found in all models. Globally deviations rarely exceed one order of magnitude. We also compare in Fig. 4 the D1M+QRPA and experimental half-lives for the much studied isotopic chains of $\mathrm{Kr}$, $\mathrm{Sr}, \mathrm{Zr}$ and Mo which are strongly deformed.

Finally, our $\beta$-decay half-lives are compared to the density function plus continuum QRPA calculation (DF3+cQRPA) of Ref. $[13,16]$ in Fig. 5 for the exotic neutron-rich nuclei along the $N=82,126$ and 184 isotones. We choose to focus on this region of the nuclear chart due to its relevance to the $r$-process nucleosynthesis [1]. Nice agreement with experimental data is found for ${ }^{130} \mathrm{Cd}$. Both the contribution of the GT and the GT plus first-forbidden (FF) transitions are given in Fig. 5 for the DF3+cQRPA calculation to illustrate the impact of the FF contributions, as predicted by [16]. Such a contribution needs to be included for some of the $N=126$ nuclei. The deviations between our results and the ones of the DF3+cQRPA approach can originate from different GT strength but also different estimates for the $Q_{\beta}$-values or reference energies $E_{0}$. We also show in the left panel of Fig. 5 the shell model predictions [17] for some of the $N=82$ nuclei that are in relatively close agreement with the DF3+cQRPA calculations and lower than ours. Such different predictions could have an impact on the production of the heavy nuclei by the r-process nucleosynthesis, but such an analysis is postponed to a future study.

\section{Conclusion}

We presented here a fully consistent pnQRPA approach using a finite-range Gogny force. We applied our model to the analysis of charge-exchange modes paying a special attention to the GT 


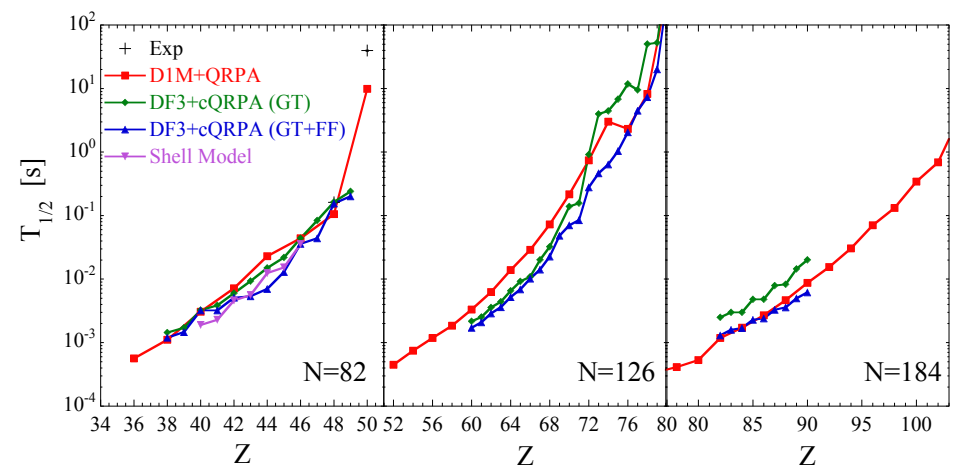

Figure 5: (Color online) Comparison between our $\beta$-decay half-life predictions and the DF3+QRPA calculation of $[13,16]$, including the GT contribution or both the GT plus FF contributions, for the neutron-rich nuclei along the $N=82,126$ and 184 isotones. For the $N=82$ isotonic chain, experimental data [15] and shell model results [17] are also shown.

resonances. The crucial role of deformation, automatically included in our approach, was analyzed. The agreement with experiment is satisfactory both for the strength distribution and the $\beta^{-}$-decay half-lives.

\section{References}

[1] M. Arnould, S. Goriely, T. Takahashi, Phys. Rep. 450, 97 (2007).

[2] P. Sarriguren and J. Pereira, Phys. Rev. C 81, 064314 (2010).

[3] M. Martini, S. Péru and S. Goriely, Phys. Rev. C 89, 044306 (2014).

[4] S. Goriely, S. Hilaire, M. Girod and S. Péru, Phys. Rev. Lett. 102, 242501 (2009).

[5] J. Dechargé and D. Gogny, Phys. Rev. C 21, 1568 (1980).

[6] S. Péru and H. Goutte, Phys. Rev. C 77, 044313 (2008).

[7] S. Péru, G. Gosselin, M. Martini, M. Dupuis, S. Hilaire et al., Phys. Rev. C 83, 014314 (2011).

[8] M. Martini, S. Péru and M. Dupuis, Phys. Rev. C 83, 034309 (2011).

[9] T. Wakasa et al.,Phys. Rev. C 55, 2909 (1997).

[10] H. Akimune et al.,Phys. Rev. C 52, 604 (1995).

[11] K. Pham et al.,Phys. Rev. C 51, 526 (1995).

[12] J. H. Thies et al., Phys. Rev. C 86, 014304 (2012).

[13] I. N. Borzov and S. Goriely, Phys. Rev. C 62, 035501 (2000).

[14] G. Audi et al.,Chinese Physics C36, 1287 (2012).

[15] G. Audi et al., Chinese Physics C36, 1157 (2012).

[16] I. N. Borzov, Phys. Rev. C 67, 025802 (2003) and I. N. Borzov, private communication (2013).

[17] G. Martinez-Pinedo and K. Langanke, Phys. Rev. Lett. 83, 4502 (1999). 